\title{
Psycholinguistic norms for action photographs in French and their relationships with spoken and written latencies
}

\author{
PATRICK BONIN, BRUNO BOYER, ALAIN MÉOT, MICHEL FAYOL, and SYLVIE DROIT \\ Université Blaise Pascal, Clermont-Ferrand, France
}

\begin{abstract}
A set of 142 photographs of actions (taken from Fiez \& Tranel, 1997) was standardized in French on name agreement, image agreement, conceptual familiarity, visual complexity, imageability, age of acquisition, and duration of the depicted actions. Objective word frequency measures were provided for the infinitive modal forms of the verbs and for the cumulative frequency of the verbal forms associated with the photographs. Statistics on the variables collected for action items were provided and compared with the statistics on the same variables collected for object items. The relationships between these variables were analyzed, and certain comparisons between the current database and other similar published databases of pictures of actions are reported. Spoken and written naming latencies were also collected for the photographs of actions, and multiple regression analyses revealed that name agreement, image agreement, and age of acquisition are the major determinants of action naming speed. Finally, certain analyses were performed to compare object and action naming times. The norms and the spoken and written naming latencies corresponding to the pictures are available on the Internet (http://www.psy.univ-bpclermont.fr/ pbonin/pbonin-eng.html) and should be of great use to researchers interested in the processing of actions.
\end{abstract}

A number of studies have attempted to identify the processes and the representations that are involved in object naming both in speaking (e.g., Barry, Hirsh, Johnston, \& Williams, 2001; Barry, Morrison, \& Ellis, 1997; Bonin, Chalard, Méot, \& Fayol, 2002; Dell'Acqua, Lotto, \& Job, 2000; Lachman, Shaffer, \& Henrikus, 1974; Vitkovitch \& Tyrrell, 1995) and in writing (Bonin et al., 2002; Bonin \& Fayol, 2000; Bonin, Fayol, \& Chalard, 2001), but less so in the latter output mode. Studies of this kind have often used a correlational/multiple regression approach. As a result of these studies, a number of important determinants of naming speed and accuracy have been identified (see below). Studies of this kind have become possible thanks to the fact that various picture characteristics and name attributes have been collected for various pictorial stimuli in different language communities and populations. It is clear that the collection of normative data on pictures has allowed for advancements in the studies designed to determine the processes and the representations involved in picture naming and, more precisely, in object naming. However, so far the majority of studies have not focused on the naming of $a c$ tions in either speaking or writing in normals. There are

The authors thank Anna Székely, Ira Bernstein, and Jonathan Vaughan for their very helpful comments on a previous version of the paper. Correspondence should be addressed to P. Bonin, Laboratoire de Psychologie Sociale et Cognitive (LAPSCO), Université Blaise Pascal, 34, avenue Carnot, 63037 Clermont-Ferrand, France (e-mail: patrick.bonin@srvpsy.univ-bpclermont.fr). only a few normative studies involving line drawings or photographs of actions (Cuetos \& Alija, 2003, in Spanish; Fiez \& Tranel, 1997; Masterson \& Druks, 1998; Székely et al., in press, in English). As far as French is concerned, we are aware of no normative study of drawings or photographs of actions. The present study attempts to fill this gap. First, we collected norms for action photographs (taken from Fiez \& Tranel, 1997) and their corresponding names in French. Second, we recorded spoken and written naming latencies in an attempt to identify certain of the determinants of action naming speed.

\section{Normative Studies Involving Pictures of Objects}

Normative studies involving pictures of objects have proven to be very useful, because researchers can use the same set of pictures across studies so that the findings can be optimally compared. In effect, before the pioneering study conducted by Snodgrass and Vanderwart (1980), researchers interested in object naming were forced to select their own pictures from different sources (e.g., clip art libraries, children's books, or dictionaries) or to ask an artist to draw the pictures. Pictures are idiosyncratic in nature and may therefore vary along a variety of dimensions. Therefore, when researchers used different pictures across studies, the results were not entirely comparable. The situation has changed in recent years since the Snodgrass and Vanderwart (1980) pictures have been standardized in different languages and for different populations (in American English in children, Cycowicz, Friedman, Rothstein, \& Snodgrass, 1997; in British En- 
glish in adults, Barry et al., 1997; and Vitkovitch \& Tyrrell, 1995; in Spanish in adults, Sanfeliu \& Fernandez, 1996; more recently in French in adults, Alario \& Ferrand, 1999; in Icelandic in adults and children, Pind, Jonsdottir, Tryggvadottir, \& Jonsson, 2000; and in Italian in adults, Dell'Acqua et al., 2000). Also, other sets of pictures of objects have been standardized (in Dutch in adults, Martein, 1995; in English in children, Berman, Friedman, Hamberger, \& Snodgrass, 1989; in French in adults, Bonin, Peereman, Malardier, Méot, \& Chalard, 2003).

Object naming studies that have adopted a multiple regression approach have found that certain characteristics of the pictures and also certain characteristics of the object names have a major impact on naming performance. Among the factors that have been investigated are name agreement, image agreement, conceptual familiarity, visual complexity, imageability, age of acquisition, and word frequency. These variables will be briefly described in turn. In addition, norms for certain of these variables have been collected for action pictures (Cuetos \& Alija, 2003; Fiez \& Tranel, 1997; Masterson \& Druks, 1998; Székely et al., in press; the present study).

Name agreement (NA) corresponds to the degree to which participants agree on a particular name to refer to a picture. Two measures of NA can be computed: the percentage of participants producing the most common name and the $H$ statistic (Snodgrass \& Vanderwart, 1980). NA has been found to be an important determinant of naming speed (Barry et al., 1997; Bonin et al., 2002; Ellis \& Morrison, 1998; Gilhooly \& Gilhooly, 1979; Lachman, 1973; Lachman et al., 1974; Paivio, Clark, Digdon, \& Bons, 1989; Snodgrass \& Yuditsky, 1996; Vitkovitch \& Tyrrell, 1995). Image agreement (IA) refers to the degree to which the mental images formed by participants in response to a picture name match the picture's appearance. Pictures that have a high IA score are produced faster in spoken (Barry et al., 1997; Bonin et al., 2002) and in written (Bonin et al., 2002) picture naming. Imageability (Imag) corresponds to the level of ease with which an object name arouses a mental image (Paivio et al., 1989). Imag has often been considered to be a reliable index of the involvement of semantic representations. Imag effects have not been found to contribute to naming times in a robust manner in picture naming (e.g., Barry et al., 1997; Ellis \& Morrison, 1998). Familiarity (Fam) refers to the familiarity of the depicted concept. This variable has been included in most picture naming regression studies, but it has not been found to contribute to naming times in a systematic and robust manner (Ellis \& Morrison, 1998; Jolicœur, 1985). Visual complexity (VC) corresponds to the number of lines and details in the drawing. Here also, VC of the pictures has not been found to contribute robustly to naming times (Barry et al., 1997; Bonin et al., 2002; Cycowicz et al., 1997; but see Ellis \& Morrison, 1998).

Age of acquisition (AoA) corresponds to the age at which a word is acquired in its spoken or written form. Most studies have used estimated AoA scores. To collect
AoA scores for words, adults are asked to indicate on a point scale the age at which they think they acquired the word in its spoken or written form. This scale consists of a number of points corresponding to different age bands. Rated and objective AoA scores have been robustly found to be reliable and strong determinants of naming speed (e.g., Barry et al., 2001; Barry et al., 1997; Bonin et al., 2002; Bonin et al., 2001; Carroll \& White, 1973; Chalard, Bonin, Méot, Boyer, \& Fayol, 2003; Ellis \& Morrison, 1998; Lachman, 1973; Lachman et al., 1974; Morrison, Chappell, \& Ellis, 1997; Morrison, Ellis, \& Quinlan, 1992; Székely et al., 2003). Although word frequency effects have been found to affect naming times (e.g., Humphreys, Riddoch, \& Quinlan, 1988; Jescheniak \& Levelt, 1994; Oldfield \& Wingfield, 1965), most past reports of word frequency effects in picture naming have not taken AoA into account. Given that AoA and word frequency are correlated variables, some researchers have cast considerable doubt on whether word frequency effects are genuine frequency effects or whether they should be attributed to AoA (Morrison et al., 1992). Nevertheless, other picture naming studies have reported significant effects of both word frequency ${ }^{1}$ and AoA (Barry et al., 1997; Ellis \& Morrison, 1998; Lachman, 1973; Lachman et al., 1974; Snodgrass \& Yuditsky, 1996; but see Barry et al., 2001; Bonin et al., 2002; Chalard et al., 2003).

\section{Normative Studies Using Photographs/Pictures of Actions}

As was already mentioned, there are only a few standardized databases corresponding to pictures of actions. To our knowledge, only four normative studies of pictures of actions have been published: three in English (Fiez \& Tranel, 1997; Masterson \& Druks, 1998; Székely et al., in press) and one in Spanish (Cuetos \& Alija, 2003). Fiez and Tranel published norms for VC, IA, NA, and Fam for 280 photographs of actions, whereas Masterson and Druks collected norms for VC, Imag, Fam, and AoA for 102 line drawings of actions. However, in these two studies, naming times for the photographs and the drawings, respectively, were not collected. Székely et al. collected naming times and norms in English for AoA, VC, and NA for a set of 275 actions taken from a variety of sources. Finally, in Spanish, Cuetos and Alija collected norms and spoken naming times for a set of 100 line drawings taken from the Masterson and Druks database.

Whereas Fiez and Tranel (1997) used photographs of actions, Masterson and Druks (1998) and Székely et al. (2003) used black-and-white drawings of actions. Historically, the pictorial stimuli used in object naming studies have taken the form of black-and-white drawings (e.g., the Snodgrass \& Vanderwart, 1980, pictures). Fiez and Tranel used color pictures and picture pairs, because their intention was to develop stimuli that most clearly depicted a broad range of actions rather than to compare performances, for instance, with black-and-white draw- 
ings of objects and photographs of actions. It might be claimed that the use of moving video pictures would be more suitable than that of photographs or drawings of action since actions are more difficult to portray than are objects. Although we acknowledge that the collection of norms for moving pictures might be useful and should be undertaken in the future, Berndt, Mitchum, Haendiges and Sandson (1997) have shown that naming performance for two different action presentation modes - namely, line drawings and moving pictures presented in video formdid not differ in aphasic patients.

As was pointed out by Sanfeliu and Fernandez (1996), language-specific norms are important since, for example, the way names are used to refer to object pictures has been shown to vary across languages. Cross-linguistic comparisons are important since they make it possible to determine whether the (correlational) structure among the variables that has been found in some languages also holds true in others. Cuetos and Alija (2003) did not compare their norms with Masterson and Druks's (1998) norms. In the Results section, certain analyses will be reported that compare the relationships found in our study with the Fiez and Tranel (1997) study, with the Masterson and Druks study, with the Székely et al. (in press) study, and with the Cuetos and Alija study. The similarities and differences between these studies and the current one will be reported in detail.

\section{Why Should We Study Action Naming?}

Picture naming is a fast and efficient process (Levelt, 1989; Levelt, Roelofs, \& Meyer, 1999). The picture naming task has been widely used by researchers involved in language production to investigate the retrieval of words from the mental lexicon-that is, lexical access. Several models have been proposed to account for lexical access in spoken picture naming (e.g., Caramazza, 1997; Dell \& O’Seaghdha, 1992; Dell, Schwartz, Martin, Saffran, \& Gagnon, 1997; Humphreys et al., 1988; Levelt et al., 1999; Roelofs, 2000). Until now, the various theoretical accounts of lexical access in language production in normals have focused on the retrieval of names from objects - that is to say, nouns. However, when producing sentences in either speaking or writing, we produce not only nouns but also verbs. Therefore, any complete model of lexical retrieval in language production should also account for how we retrieve verbs from the mental lexicon.

If we assume that action naming involves levels of processing similar to those that have been identified in object naming, we should find that the factors that contribute to naming latencies in object naming are very similar to those that reliably contribute to naming latencies in action naming (see below). However, the literature on verb processing suggests that certain differences between object and action naming might be anticipated. From an empirical point of view, the identification of the variables that reliably contribute to action naming speed is important because it will help researchers identify the factors that need to be controlled for when designing experiments on action naming.

Verbs not only belong to a different grammatical category than nouns, but they also differ on other dimensions. Verbs are more difficult to remember than are nouns and are acquired later in life (Gentner, 1981; but on the latter point, see Tardif, 1996). The meanings of verbs are sparser than are the meanings of nouns. Moreover, verbs are numerous for very high and high word frequency values but less numerous for lower word frequency values (Gentner, 1981). In contrast, nouns are more numerous in the lower word frequency range (Gentner, 1981). Imag scores have been found to be lower for nouns than for verbs, even when the nouns and the verbs are pictoriable (Masterson \& Druks, 1998). In cognitive neuropsychology, double dissociations between noun and verb processing have been reported in patients (e.g., Caramazza \& Hillis, 1991; Denes \& Dalla Barba, 1998; McCarthy \& Warrington, 1985; Miceli, Silveri, Nocentini, \& Caramazza, 1988). For instance, Caramazza and Hillis have described a double dissociation between nouns and verbs in spoken and in written production. Although the two patients they studied had no comprehension difficulties for either nouns or verbs, one patient performed better on nouns than on verbs in writing tasks (spelling to dictation and written naming), whereas the other patient exhibited the opposite pattern of performance-that is, he performed better on nouns than on verbs in spoken production tasks (word reading and spoken naming). According to Shapiro and Caramazza $(2001 \mathrm{a}, 2001 \mathrm{~b})$, the verb and noun grammatical categories are represented in the output phonological and orthographic lexicon, respectively. However, there is some discussion as to whether the reported dissociations between nouns and verbs can be considered to be genuine grammatical dissociations or whether they can be explained by different semantic characteristics corresponding to nouns and verbs (Bird, Howard, \& Franklin, 2001; Shapiro \& Caramazza, 2001a, 2001b). According to the latter account, the dissociations between nouns and verbs should not be ascribed to a lexical but to a semantic level. Finally, neuroimaging and electrophysiological data have suggested that nouns and verbs activate different parts of the brain (e.g., Damasio \& Tranel, 1993; Daniele, Giustolisi, Silveri, Colosimo, \& Gainotti, 1994; Molfese, Burger-Judisch, Gill, Golinkoff, \& HirschPasek, 1996). Thus, certain differences in the processing of verbs and nouns can be anticipated. However, we still need to determine precisely the level(s) at which the processing differences are located. Since some studies suggest that the semantic representations corresponding to actions are sparser than those corresponding to objects (Gentner, 1981; Masterson \& Druks, 1998), Imag — a variable recognized as indexing semantic representationsshould make a greater contribution in action naming than in object naming. Given also that the way actions are lexicalized is more variable than the way objects are lexicalized (Gentner, 1981), NA should play a greater role in 
action naming than in object naming. Finally, one issue that needs to be addressed is whether the grammatical category-nouns or verbs-makes a specific contribution in naming speed beyond that of other variables as one would predict if the noun and verb grammatical categories were organizational dimensions of the mental lexicon (Shapiro \& Caramazza, 2001b) and as, indeed, the Székely et al. (in press) study, reported below, suggests.

It is clear that a necessary first step for the investigation of potential differences between action and object naming is to collect norms for pictures of actions and naming times for the pictures. Indeed, Cuetos and Alija (2003) have shown that AoA and NA are reliable determinants of spoken action naming speed in normal adults. Cuetos and Alija also compared action naming speed with object naming speed in participants taken from the same pool (Cuetos, Ellis, \& Alvarez, 1999). They found that (1) NA was more important in verb naming than in object naming, whereas the reverse was true regarding word frequency and Fam, and (2) naming times were longer for action naming than for object naming. According to these authors, the latter result suggests that actions are more difficult to process because there is usually more happening in action pictures than in object pictures, or because verbs have less accurate representations in the lexicon than do nouns, or even because verbs elicit more competition between alternative responses than do objects. Székely et al. (in press) have also compared the factors affecting object and action naming in timed picture naming tasks in English-speaking adults. The various analyses they conducted led them to conclude that action naming is more difficult than object naming even though verbs tend to be shorter and of higher frequency than are nouns. They also found that action pictures are more visually complex than are object pictures, that verbs are acquired later than are nouns, and that verbs are more often shared by two or more pictures in the stimulus set. In a multiple regression analysis that took into account several predictors (e.g., AoA, VC, NA, etc.) including a dichotomous action-object variable, they found that word length, objective AoA, word complexity, and NA made reliable independent contributions in predicting naming latencies. Importantly, the action-object variable also made an independent and reliable contribution. The latter finding strongly suggests that action naming is still much more difficult than object naming is, even though several factors that may potentially account for the naming difficulty difference between nouns and verbs (in particular, NA and VC) are statistically controlled for. Given that Cuetos and Alija's study and Székely et al.'s study are, to the best of our knowledge, the only studies that have collected naming data for action pictures, the generalizability of these findings must be determined. Indeed, in the present study, we investigated some of the determinants of action naming speed both in speaking and in writing. On the basis of the Cuetos and Alija and the Székely et al. studies, we anticipated that
NA and AoA would be reliable determinants of action naming latencies in both production modes. In the light of the findings obtained by Székely et al., we report in the final part of this article certain analyses that were designed to compare the difficulty of naming actions versus objects - as indexed by naming times-on the basis of the Bonin et al. (2002) object naming study and the present action naming study.

To summarize, the normative study reported here will be useful to researchers who are, more generally, interested in action processing. This article is organized into two parts. The first part will describe the collection of norms in French-speaking adults for action photographs taken from Fiez and Tranel's (1997) study. The photographs were standardized on NA, IA, Fam, VC, Imag, AoA, and the duration of the actions. The norms are available on the Internet at http://www.psy.univ-bpclermont. fr/ pbonin/pbonin-eng.html. The statistics corresponding to the variables collected for action items will also be reported and compared with the same variables collected for object items in French. Correlational analyses were performed on the variables collected for actions, and the results of these analyses were compared with the results reported by Fiez and Tranel (1997), Masterson and Druks (1998), and Székely et al. (in press) in English and Cuetos and Alija (2003) in Spanish. The second part will report the collection of spoken and written naming times corresponding to the standardized photographs (also available on the Internet from the above-mentioned URL) and certain comparisons between action and object naming times. The action naming times will be very useful when selecting materials for designing experiments.

\section{COLLECTION OF THE NORMS}

\section{Method}

Participants. A total of 180 psychology students from Blaise Pascal University participated in the normative study in return for course credits. Different participants were involved in each of the six rating tasks (30 participants in each task). The six tasks were NA, IA, Fam, VC, AoA and Imag. The participants (17 males and 163 females; mean age, 20.52 years; range, 17-38 years) were all native speakers of French and had normal or corrected-to-normal vision. All the separate rating tasks were performed collectively.

Material. The initial stimuli were the 280 photographs depicting actions and events from the Fiez and Tranel (1997) battery. The preliminary selection was performed using only single pictures depicting persons, animals, and objects engaged in ongoing actions. Thus, the picture pairs that were used by Fiez and Tranel to depict a change of state or an event were not used since our goal was to provide naming times for single pictures. Next, a total of 210 photographs were shown individually to 10 participants (not involved in the normative study) who had to assign a verb to each action depicted by the photographs. Sixty-eight pictures were discarded because either (1) they had a very low NA score $(<30 \%)$ or $(2)$ they led to the production of the same modal verb in French (e.g., in French the infinitive form pousser is used for the three actions to push, to shove, and to grow). In the latter case, the picture with the highest NA score was selected (e.g., to push for pousser). Thus, the final pool of pictures retained for inclusion in the normative study was 142 . 
Procedure. The participants were tested collectively. The procedures used by Fiez and Tranel (1997) to collect the norms were closely adhered to. At the beginning of each rating task, the instructions were provided both in writing on a separate sheet of paper and verbally by the experimenter. The participants were instructed to perform their assigned rating task carefully and consistently. Individual answer sheets were prepared for each rating task. In each rating task, short breaks were given to the participants. For the NA, IA, Fam, and VC tasks, the pictures were projected onto a large white screen by means of an overhead projector. For the AoA and Imag rating tasks, the written modal verb taken from the NA task corresponding to the action was provided on the individual answer sheets. For all the rating tasks except NA, 5-point scales were used. Great care was taken to explain to the participants that the full range of scale values had to be employed and not only the extremes.

In the NA task, the participants were told to look carefully at each picture and to write down on the answer sheet a single word (the infinitive form of a verb) that best described what the person, animal, or object was doing. Each time the participants failed to name a picture, they had to indicate whether it was because they did not know the action (they were instructed to write down "DKA"), they did not know the name of the action ("DKN"), or they were in a tip-ofthe-tongue state ("TOT"). A preliminary test on a subset of pictures with different participants revealed that $7 \mathrm{sec}$ was enough to complete a written response. Therefore, each picture was presented for $7 \mathrm{sec}$.

In the IA task, the participants were asked to judge how closely each photograph resembled their mental image of the action. To this end, the experimenter spoke aloud the modal name corresponding to the picture prior to its presentation, waited approximately $5 \mathrm{sec}$, and then presented the picture on the screen. During this 5-sec period, the participants had to generate a mental image corresponding to the modal verb spoken aloud while keeping their eyes closed or looking at the black screen. Once the photograph was displayed, they rated on a 5-point scale the degree of agreement between the photograph and their generated mental image, with 1 corresponding to low agreement and 5 corresponding to high agreement.

In the Fam task, the participants were asked to evaluate the familiarity of the action depicted by each picture in terms of how usual or unusual the action was in their realm of experience. Familiarity was defined as "the degree to which you come into contact with or think about the action." Care was taken to explain to the participants that the rating had to be attributed to the action itself and not to the way in which it was photographed or the particular object used to demonstrate the action. A 5-point scale was used, with 1 corresponding to a very unfamiliar action and 5 corresponding to a very familiar action.

In the VC task, the participants were told to rate the visual complexity of each photograph. They were told to rate the complexity of the photograph itself rather than the complexity of the real-life action that it represented. The score of 5 corresponded to very complex and the score of 1 to very simple. The visual complexity was defined as the number of details or the intricacy of the lines in the photograph.

For the Imag, AoA, and duration tasks, the ratings were performed on the basis of the written modal verbs that were taken from the individual answers from the NA task. A booklet containing all the modal verbs was prepared. A 5-point scale was printed below each modal verb. In the Imag task, the participants had to indicate on a 5-point scale whether the verb evoked a mental image with very great difficulty (rated 1) down to very easily (rated 5). In the AoA tasks, the participants had to estimate the age at which they thought they had learned each of the verbs in its written or oral form. For this task, the values of the scale corresponded to 3-year age bands, with 1 corresponding to learned between $0-3$ years and 5 corresponding to learned at age 12 or after. In the duration task, the participants had to estimate from the modal verb the time it takes to perform the corresponding action, with 1 corresponding to very short action and 5 corresponding to very long action. We included this variable because the temporal dimension of actions might be relevant when naming actions from photographs. If the time taken to physically perform an action is mentally encoded, the temporal dimension of the action might come into play when naming the action from a photograph. Therefore, the time it takes to perform an action might be translated into naming times.

The written word frequency and the cumulative frequency values (i.e., Frantext frequency measures taken from the LEXIQUE database, New, Pallier, Ferrand, \& Matos, 2001) for the modal verbs are also provided when available. The word frequency values are based on a corpus of 31 million words. Given that certain lexical processing models assume morphological decomposition, we also provide the cumulative frequency of the forms corresponding to the modal verbs. The abbreviation NA is used to indicate that the value was not available in LEXIQUE (New et al., 2001).

\section{COLLECTION OF THE NAMING TIMES}

\section{Method}

Participants. A total of 60 undergraduate students ( 55 females and 5 males; mean age, 20.17 years; range, 17-28 years) from Blaise Pascal University (Clermont-Ferrand) participated in the experiment in order to fulfill a course requirement and were given course credits. All were native speakers of French with normal or corrected-tonormal vision. Half of the participants were randomly assigned to the spoken action naming task and the remaining half to the written action naming task. None of them had participated in the six rating tasks.

Material. The stimuli consisted of the 142 photographs used in the rating tasks. Ten additional action pictures were used for training.

Apparatus. The experiment was run using PsyScope (Cohen, MacWhinney, Flatt, \& Provost, 1993) on a PowerMacintosh. The computer controlled the presentation of the pictures and recorded the latencies. A graphic tablet (WACOM UltraPad A5) and a contact pen (SP-401) were used to record the graphic latencies. An AIWA CM-T6 small tie-pin microphone connected to a buttonbox was used to record the spoken latencies. The recording accuracy for the latencies was to the nearest millisecond.

Procedure. The participants were tested individually and were randomly assigned to either the written or spoken action naming task.

Spoken action naming. The participants sat in front of the screen at a distance of about $60 \mathrm{~cm}$. They were told that they would have to say aloud the infinitive form of the verb corresponding to any given action photograph presented on the screen as quickly and as accurately as possible, and to avoid saying "um" or "er" before a verb. Each time the participants failed to name a photograph, they had to indicate whether it was because they did not recognize the action (they had to say aloud "DKA") or because they did not know the name of the action ("DKN"). However, when the participants felt they knew its name but were not able to retrieve it immediately, they had to say aloud "tip of the tongue" (TOT). The experimenter monitored the participants' responses and scored them for correctness.

Each trial had the following structure: A ready signal (*) appeared on the screen for $500 \mathrm{msec}$ and was immediately followed by the picture. The next trial began $5,000 \mathrm{msec}$ after the participants initiated their response. This intertrial delay was established on the basis of similar studies (Bonin et al., 2002; Bonin et al., 2001). A short break was given to the participants after every 50 trials. The experiment started with 10 practice pictures. The order of presentation was randomized for each participant. The experimenter recorded all naming errors, hesitations, and voice key failures. The entire session lasted about $45 \mathrm{~min}$.

Written action naming. The procedure was the same as that for spoken action naming except that the participants had to write down the verb (in the infinitive form) corresponding to each presented 
photograph as quickly and as accurately as possible. The written responses were timed as follows: The participants sat with the stylus right above the tablet so that the latency was the time taken to make contact after picture onset. In order to avoid any variability in the positioning of the stylus before each word was written, a line was drawn and the participant was obliged to position the stylus directly above the start of the line. More precisely, we prepared response sheets (size, $21 \times 29.7 \mathrm{~cm}$ ) to enable us to gather all the written responses relating to the different verbs. These response sheets consisted of five columns of 20 lines each, with the different lines drawn one above the other at a constant interval of $0.7 \mathrm{~cm}$. All of the lines were $3.5 \mathrm{~cm}$ long. The experimenter systematically ensured that the instructions were adhered to and always corrected the participants if they failed to follow them. Also, they were instructed to write down either "DKA," "DKN," or "TOT" when the name was not immediately available. A short break was given to the participants after every 50 trials. As in the spoken naming condition, the order of presentation was randomized, and 10 pictures were used for initial practice. The entire session lasted about $45 \mathrm{~min}$.

\section{RESULTS AND DISCUSSION}

\section{Rating Data}

The mean ratings collected for each action photograph are available electronically on the Internet (at the previously mentioned URL). The items are listed alphabetically. For each action photograph, the following information is provided: (1) the number of the photograph used in the Fiez and Tranel (1997) database (the photographs can be obtained electronically on request from these authors); (2) the most common (modal) verb obtained in the untimed NA task (together with both the intended verb in parentheses whenever it was different from the modal name and an approximate English translation of the modal verb); (3) two measures of NA corresponding to the percentage of participants giving the most common verb and the $H$ statistic ${ }^{2}$ and (4) the means and the standard deviations. The word frequency value of the infinitive form of the modal verb and the cumulative word frequency value of the modal verb (taken from New et al., 2001) are also provided whenever available (as already stated, the abbreviation NA is used when frequency values were not available in LEXIQUE).

The various nonmodal verbs and their corresponding frequencies of occurrence are also provided for each of the photographs. The number of naming failures (DKN, DKA, and TOT) is also given for each item. Finally, the mean spoken and written naming latency for each item is given in milliseconds.

\section{Descriptive Statistics}

Descriptive statistics for the different variables are presented in Table 1.

As was already stated, two measures of NA were computed: the $H$ statistic and the percentage of participants producing the modal verb (\%NA). The $H$ value equals 0 when the modal verb was provided by all participants. Higher $H$ values indicate lower levels of name agreement.

In comparison with two databases corresponding to pictures of objects, which have recently been standardized in French (i.e., Alario \& Ferrand, 1999; Bonin, Méot, et al., 2003) on the same variables as those used in the present study except duration, the NA percentage for actions was lower (and $H$ conversely higher) on the measures of central tendency. We subdivided the frequency distributions of the NA scores into 10-unit intervals for both objects (NA scores taken from Alario \& Ferrand, 1999) and actions. As is shown in Figure 1, the NA scores exhibited reliably different frequency distributions $\left[\chi^{2}(8)=48.71, p<.001\right] .^{3}$

As can be seen from Figure 1, there are more objects than actions for which the NA score was $100 \%(58.90 \%$ vs. $28.20 \%$ ), whereas for all the other NA bands the frequencies were higher for actions than for objects.

The \%NA and $H$ variables appeared to be less skewed for actions than for objects, and the skews acted in op-

Table 1

Summary Statistics in the Current Sample

\begin{tabular}{|c|c|c|c|c|c|c|c|c|c|c|}
\hline & $\begin{array}{l}\text { NA } \\
(H)\end{array}$ & $\begin{array}{l}\text { NA } \\
(\%)\end{array}$ & IA & Fam & $\mathrm{VC}$ & Imag & AoA & Dur & $\begin{array}{l}\text { Word } \\
\text { Freq. }\end{array}$ & $\begin{array}{l}\text { Cum. } \\
\text { Freq. }\end{array}$ \\
\hline$M$ & 0.96 & 73.00 & 3.53 & 2.80 & 2.35 & 3.99 & 2.36 & 2.61 & 19.56 & 111.38 \\
\hline$S D$ & 0.72 & 21.51 & 0.87 & 1.02 & 0.82 & 0.54 & 0.66 & 1.00 & 34.15 & 173.15 \\
\hline Median & 0.93 & 73.33 & 3.73 & 2.72 & 2.05 & 4.05 & 2.37 & 2.41 & 8.06 & 47.29 \\
\hline Range & 3.01 & 90.00 & 3.48 & 3.67 & 3.32 & 2.63 & 3.13 & 3.64 & 234.71 & 960.65 \\
\hline Min & 0.00 & 10.00 & 1.39 & 1.27 & 1.14 & 2.34 & 1.10 & 1.05 & 0.10 & 0.29 \\
\hline Max & 3.01 & 100.00 & 4.88 & 4.93 & 4.46 & 4.97 & 4.23 & 4.68 & 234.81 & 960.94 \\
\hline Q1 & 0.24 & 60.00 & 2.88 & 1.93 & 1.79 & 3.66 & 1.87 & 1.76 & 2.03 & 17.38 \\
\hline Q3 & 1.49 & 93.33 & 4.27 & 3.52 & 2.84 & 4.38 & 2.83 & 3.33 & 20.76 & 114.47 \\
\hline IRQ & 1.25 & 33.33 & 1.39 & 1.59 & 1.05 & 0.72 & 0.96 & 1.57 & 18.73 & 97.09 \\
\hline Skew & 0.81 & 1.50 & 0.64 & 1.01 & 3.04 & 0.85 & 0.92 & 1.41 & 2.11 & 2.25 \\
\hline
\end{tabular}

Note-NA, name agreement $(H$, statistic $H$; \%, percentage of participants giving the most common verb); IA, image agreement; Fam, conceptual familiarity; VC, visual complexity; Imag, imageability; AoA, age of acquisition; Dur, duration; Word Freq., word frequency of the infinitive form of the modal verb from LEXIQUE (Frantext; New et al., 2001); Cum. Freq., cumulative word frequency of the modal verb from LEXIQUE (Frantext; New et al., 2001); $M$, mean; SD, standard deviation; Q1, 25th percentile; Q3, 75th percentile; IRQ, interquartile range; Skew, (Q3-median)/(median-Q1): $>1$ is positively skewed. 


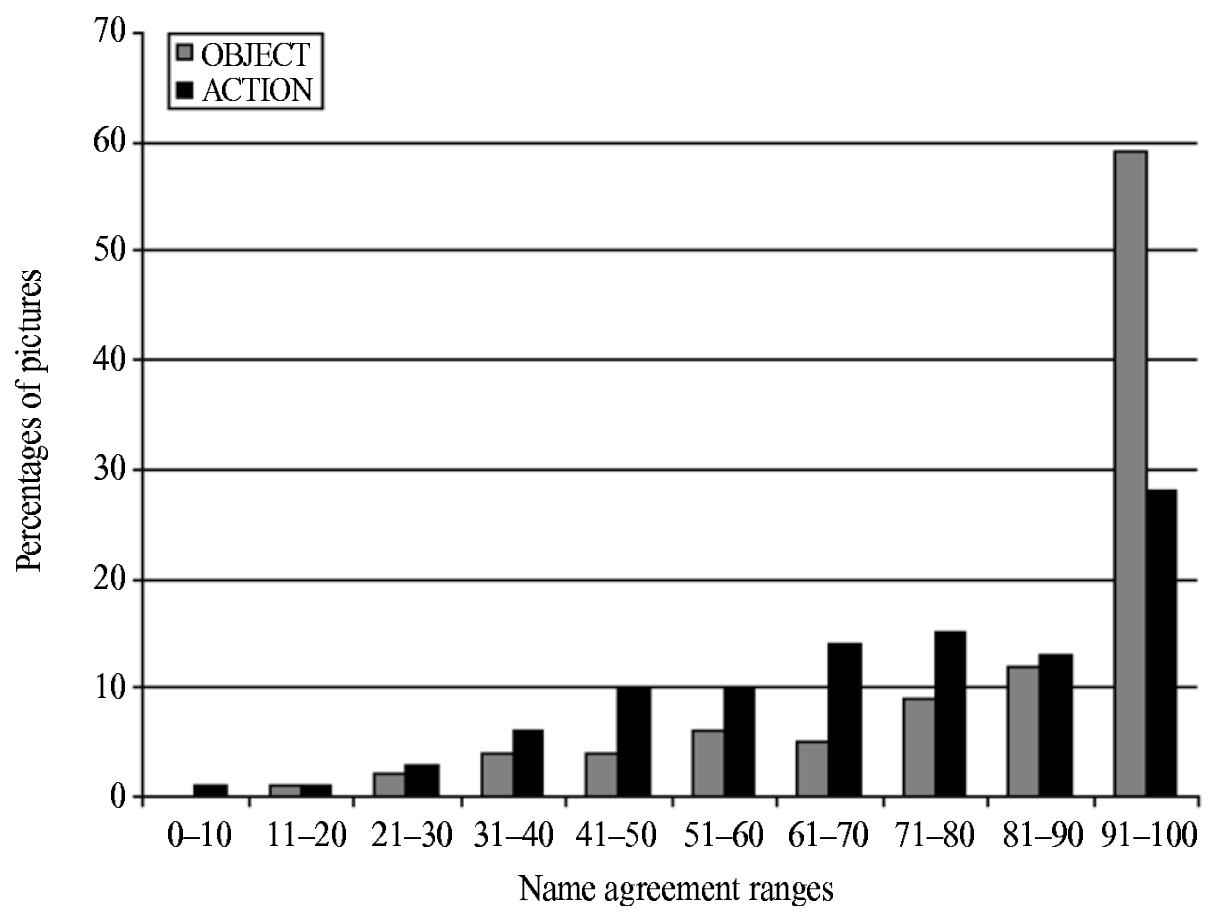

Figure 1. Distribution of pictures (in percentages) of objects and actions as a function of name agreement ranges.

posite directions. However, it appeared that the index used to measure the skew was not sufficiently sensitive because the forms of the distributions were clearly asymmetric (positively skewed for $H$ and negatively skewed for $\% \mathrm{NA}$ ), but with a long flat portion of the distribution occurring after (for \% NA) or before (for $H$ ) the tail. Fisher's $g_{1}$ coefficient revealed that the asymmetry was, in fact, in the same direction but was less pronounced for actions than for objects. For instance, concerning the $H$ measure, the corresponding values were $g_{1}=.421$ for actions and $g_{1}=.774$ for objects. The number of alternative names was higher for actions than for objects (3.75 vs. 2.23). The descriptive statistics for IA and Fam for actions were very similar to those observed for objects. As far as VC is concerned, the central tendency measures were lower in magnitude for the actions than for the objects. Also, $\mathrm{VC}$ resulted in a large positive skew for the actions, whereas no skew was observed for the objects. The rated AoA values of the modal verbs were somewhat lower on the central tendency measures than those of the nouns. The word frequency characteristics (e.g., means, standard deviations, etc.) of the modal verbs were similar to those observed for the nouns, except that the frequency range was higher for the nouns than for the verbs. Imag norms have recently been collected in French for 866 object names including those used in the Alario and Ferrand (1999) study and those used in the Bonin, Peereman, et al. (2003) study (Bonin, Meot, et al., 2003). Descriptive statistics on Imag scores were very similar for actions and objects, with the exception that, for both for the quartiles and the mean, Imag values were somewhat higher for objects than for actions. Also, the negative skew was larger for objects than for actions.

A series of analyses of variance was performed with nature of the items (action vs. object) taken as a factor on the means corresponding to NA (\%NA and $H$ ), IA, Fam, VC, Imag, word frequency (log transformed), rated AoA, and also the number of alternative names. ${ }^{4}$ These analyses confirm the descriptions reported above: There were significant differences between actions and objects on NA (\% NA and $H$ ), VC, Imag, rated AoA, and number of alternative names. The results are in agreement with those obtained by Székely et al. (in press) regarding NA and the number of alternative names in that actions yield fewer high agreement scores than do objects and also give rise to more different alternative names than do objects. However, there are also certain interesting differences. Contrary to Székely et al., we did not find that action modal names are higher in frequency than are object modal names. Also, we did not observe that the VC of the actions was higher than that of the objects, in contrast to Székely et al.'s finding. It is worth noting that the measures of VC were objective measures in Székely et al.'s study, whereas we used a scaled measure of VC. Moreover, whereas the action pictures used in the Székely et al. study were black-and-white drawings, the action pictures used in the present study were colored photographs of actions. Maybe the difference in the format used to depict 
the actions and the way in which VC is measured could account for the discrepancy.

\section{Correlational Analyses}

The correlations obtained on the set of variables are shown in Table 2. The two measures of frequency were log transformed.

As was expected, the correlation between the two NA measures ( $H$ and \% NA) was high and negative, and the correlation between the frequency of the infinitive modal form of the verb and the cumulative frequency of the verbal form was high and positive.

AoA was significantly correlated with all variables except VC. Thus, this result suggests that early-acquired verbs have higher name agreement scores, refer to more familiar actions, generate mental images of the corresponding action more easily, and are also more frequent than are late-acquired verbs.

The negative correlation between Fam and VC indicates that familiar actions are depicted by photographs that are less visually complex (or the reverse), whereas the positive correlations between Fam and the two measures of word frequency suggest that more frequent verbs are related to more familiar actions (and also the reverse).

NA (\%NA and $H$ ) was significantly correlated (positively and negatively, respectively) with both IA and Imag. The positive correlation between NA and IA suggests that high NA scores are given to photographs for which there is a good match between the mental images that are generated from the modal verb and the action depicted by the photograph. The positive correlation between NA and Imag indicates that high name agreement scores tend to be given to photographs for which the mental images are easier to generate from the corresponding modal verb.

The correlation between duration and VC was positive and reliable, thus indicating that the actions for which the duration is estimated as long are associated with more visually complex photographs (and also the reverse).

Finally, correlations were observed both between AoA and IA (positive) and between word frequency and IA (negative). These correlations indicate that high IA scores are more strongly associated with late-acquired and low frequency verbs than with early-acquired and high frequency verbs.

\section{Factor Analysis}

In order to obtain further information on the main multivariable structures of our normative database, a principal component factor analysis and a varimax rotation was performed. Only the $H$ statistic for NA was considered in the analyses because (1) the heterogeneity of the verbs provided for a photograph is better indexed by the $H$ measure than by \%NA (Snodgrass \& Vanderwart, 1980) and (2) using both NA measures would have given too much weight to the NA variable. Also, we used only the cumulative word frequency (log transformed) measures because the word frequency measures corresponding to the infinitive verbal form might underestimate the frequency of the verbal forms.

Two factors were retained because the scree plot showed no obvious break after the first eigenvalue, and the third eigenvalue was equal only to 1.24 . The two factors accounted for $51.18 \%$ of the variance. Table 3 shows the loadings of the variables. Factor 1 essentially loads on AoA and VC, which are opposed to word frequency and Fam. Given the correlations between duration and this set of variables (and more particularly VC), duration was also partly expressed on this factor. The second factor loads on IA and Imag, which are opposed to NA.

\section{Comparison With the Fiez and Tranel (1997), Masterson and Druks (1998), Székely et al. (in press), and Cuetos and Alija (2003) Databases}

Cross-linguistic comparisons are important since they make it possible to determine whether the (correlational)

Table 2

Correlations Among the Measured Variables

\begin{tabular}{|c|c|c|c|c|c|c|c|c|c|}
\hline & $\begin{array}{l}\text { NA } \\
(H) \\
\end{array}$ & $\begin{array}{l}\text { NA } \\
(\%) \\
\end{array}$ & IA & Fam & $\mathrm{VC}$ & Imag & AoA & Dur & $\begin{array}{l}\text { Word } \\
\text { Freq. }\end{array}$ \\
\hline $\mathrm{NA}(\%)$ & $-.837 *$ & & & & & & & & \\
\hline IA & $-.332 *$ & $.328 *$ & & & & & & & \\
\hline Fam & -.104 & .215 & .051 & & & & & & \\
\hline $\mathrm{VC}$ & .056 & -.110 & -.021 & $-.510 *$ & & & & & \\
\hline Imag & $-.249^{*}$ & $.253^{*}$ & .140 & .215 & -.016 & & & & \\
\hline AoA & $.226^{*}$ & $-.258 *$ & $.301 *$ & $-.391 *$ & .203 & $-.346^{*}$ & & & \\
\hline Dur & -.075 & -.030 & .194 & $-.266^{*}$ & $.453 *$ & .033 & $.216^{*}$ & & \\
\hline Word Freq. & -.142 & .215 & $-.277^{*}$ & $.310 *$ & -.176 & .049 & $-.647 *$ & -.137 & \\
\hline Cum. Freq & -.138 & $.217 *$ & $-.244^{*}$ & $.351 *$ & $-.249 *$ & -.050 & $-.607 *$ & -.205 & $.893 *$ \\
\hline
\end{tabular}

Note-NA, name agreement $(H$, statistic $H ; \%$, percentage of participants giving the most common verb); IA, image agreement; Fam, conceptual familiarity; VC, visual complexity; Imag, imageability; AoA, age of acquisition; Dur, duration; Word Freq, word frequency of the infinitive form of the modal verb from LEXIQUE (Frantext; New et al., 2001); Cum. Freq., cumulative word frequency of the modal verb from LEXIQUE (Frantext; New et al., 2001). $* p<.01$. 
Table 3

Factor Analysis (Varimax Rotation)

\begin{tabular}{lrr}
\hline Variable/Factor & 1 & \multicolumn{1}{c}{2} \\
\hline NA $(H)$ & .124 & -.747 \\
IA & .325 & .671 \\
Fam & -.696 & .257 \\
VC & .640 & -.026 \\
Imag & -.184 & .643 \\
AoA & .761 & -.180 \\
Dur & .583 & .282 \\
Cum. Freq. $(\log )$ & -.723 & -.055 \\
\hline
\end{tabular}

Note-NA, name agreement $(H$, statistic $H)$; IA, image agreement; Fam, conceptual familiarity; VC, visual complexity; Imag, imageability; AoA, age of acquisition; Dur, duration; Cum. Freq., cumulative word frequency of the modal verb from LEXIQUE (Frantext; New et al., 2001).

structure among the variables that has been found in some languages also holds true in others. The following analyses were therefore performed to compare the relationships found in our database with four databases available for action pictures: Fiez and Tranel (1997), Masterson and Druks (1998), Székely et al. (in press), and Cuetos and Alija (2003).

Fiez and Tranel (1997). Fiez and Tranel's (1997) NA scores were very similar to those found in the current database. The same holds true as far as VC is concerned. However, the mean Fam and IA scores were higher (and less heterogeneous) in the Fiez and Tranel database than in the present one. Finally, the mean cumulative frequency was higher and the scores were more heterogeneous in our database than in Fiez and Tranel's.

All the correlations reported by Fiez and Tranel (1997) had the same signs as those observed here. The correlations between Fam and NA and between IA and Fam were higher in Fiez and Tranel's database than in the present one.

Masterson and Druks (1998). The mean cumulative word frequency was lower in the Masterson and Druks (1998) database than in the present one. Also, the frequency values were less variable in the Masterson and Druks database than in the present one. Because the scales used by Masterson and Druks were different from those used here, no comparison was possible concerning the measures of central tendency or of the dispersion of the other variables.

The signs of the correlations reported by Masterson and Druks (1998) were the same as those observed in our database. However, in absolute terms, AoA was more highly correlated with word frequency and less so with familiarity in our database than in the Masterson and Druks database.

Székely et al. (in press). Only two kinds of measures are common to the Székely et al. (in press) database and ours: \%NA (and the $H$ statistic) and word frequency. The $\%$ NA scores were very similar for both the means and the variances. The mean of the $H$ statistic was slightly higher in the Székely et al. database than in the present one. The mean log frequency was much lower in our database than in Székely et al.'s. The correlations be- tween these three variables were very similar in the two databases.

Cuetos and Alija (2003). In contrast to what has been noted concerning the English and American-English databases, the mean cumulative frequency was higher in Spanish than in French. Since Cuetos and Alija (2003) used the same scales as those used by Masterson and Druks (1998), which are different from those used here, it was not possible to compare our results on the other variables.

The sign of the correlation between VC and cumulative frequency was opposite in French (-.249) and Spanish (.124, n.s.). The correlation between VC and Fam was not significant in Spanish (-.099), whereas it was large and reliable in French $(-.51)$. The correlations between the two measures of word frequency (form frequency and cumulative value, respectively) and Fam were lower in French (.31 and .351) than in Spanish (.548 and .426), whereas the correlations between the two measures of word frequency and AoA were larger in French $(-.647$ and -.607$)$ than in Spanish $(-.453$ and -.437$)$.

\section{Real-Time Naming Data}

Spoken and written naming latencies were trimmed on the basis of item rather than participant means since item difficulty is such a large source of variance in naming time (Snodgrass \& Yuditsky, 1996). Trimming was accomplished by eliminating all latencies greater than two standard deviations from each item's mean.

Multiple regression analyses were performed with spoken and written naming latencies included respectively as the dependent variables and NA, IA, Imag, VC, Fam, AoA, cumulative frequency (log transformed), and duration as the independent variables. A word length variable was also included (i.e., number of phonemes and letters in spoken and written naming, respectively).

As has generally been the case in object naming studies using multiple regression analyses (e.g., Barry et al., 1997; Bonin et al., 2002), items having a very low NA score were not considered. More specifically, only items with an NA of at least $50 \%$ (taken from the norming study) were considered..$^{5}$ Analyses were thus performed on 106 (spoken naming) and 107 (written naming) items.

Table 4 reports the results of simple correlational analyses between written and spoken naming latencies and each of the other variables. Naming latencies were correlated with NA, IA, and Imag. Moreover, spoken latencies were positively and significantly correlated with both AoA and Fam, whereas written latencies were significantly correlated with word length. Cumulative frequency was not significantly correlated with naming latencies in either task.

Table 5 reports the results from the multiple regression analyses in spoken and written picture naming.

In both tasks, NA and IA were significant determinants of naming latencies. AoA was also a reliable determinant of naming latencies in both tasks. A reliable contribution of word length was found in written naming only. The results are in agreement with Cuetos and Alija's (2003) and Székely et al.'s (in press) studies, which 
Table 4

Correlations Between Naming Times and the Other Measured Variables

\begin{tabular}{lcc}
\hline Variable & SL & WL \\
\hline NA $(H)$ & $.439^{*}$ & $.516^{*}$ \\
IA & $-.490^{*}$ & $-.549^{*}$ \\
Fam & $-.307^{*}$ & -.197 \\
VC & .223 & .135 \\
Imag & $-.270^{*}$ & $-.336^{*}$ \\
AoA & $.226^{*}$ & .097 \\
Dur & -.003 & -.149 \\
Cum. Freq. & -.124 & .007 \\
Phons & -.006 & $.256^{*}$ \\
Let & & \\
\hline
\end{tabular}

Note-SL, spoken latency; WL, written latency; NA, name agreement ( $H$, statistic $H$ ); IA, image agreement; Fam, conceptual familiarity; VC, visual complexity; Imag, imageability; AoA, estimated age of acquisition; Dur, duration; Cum. Freq., cumulative word frequency (log transformed) of the modal verb from LEXIQUE (Frantext; New et al., 2001); Phons, number of phonemes; Let, number of letters. $\quad * p<.01$.

also found that AoA and NA are reliable determinants of action naming speed in Spanish and English when drawings of actions are used. Given that Barry et al. (1997) found that AoA and word frequency interact, a regression analysis was performed with the inclusion of the interaction term, but it turned out to be not significant.

A multiple regression analysis performed on correct naming rates yielded exactly the same pattern of results as that found for the latencies. In both spoken and written naming, NA, IA, and AoA were reliable determinants of correct naming rates.

Finally, for both spoken and written naming, simultaneous regression analyses were performed with naming latencies as the dependent variable and the two factors extracted using the principal component factor analysis described above as the independent variables. These analyses revealed that the first factor was significant in spoken naming only, whereas the second factor was significant in both spoken and written naming. As far as spoken naming is concerned, the amount of unique variance accounted for by the second factor was about five times more than that accounted for by the first factor. These results are in agreement with those obtained in the multiple regression analyses described above.

In general, the results of the multiple regression analyses are consistent with those reported in the literature on object naming (e.g., Bonin et al., 2002; Chalard et al., 2003). In effect, in line with previous studies, we found that NA is an important determinant of naming speed (Barry et al., 1997; Bonin et al., 2002; Ellis \& Morrison, 1998; Gilhooly \& Gilhooly, 1979; Lachman, 1973; Lachman et al., 1974; Paivio et al., 1989; Snodgrass \& Yuditsky, 1996; Vitkovitch \& Tyrrell, 1995). The finding of a reliable effect of NA was clearly anticipated for action naming given that the way actions are lexicalized is more variable than the way objects are lexicalized (Gentner, 1981). IA has not previously been very frequently included as a predictor of naming speed in object naming studies, but it also happens to be a significant determinant of action naming latencies, as was found by Barry et al. (1997) and by Bonin et al. (2002) for object naming.

The finding of a reliable contribution of AoA in action naming adds further evidence for the claim that lexical representations-here, the lexical representations corresponding to actions - are accessed faster when words are acquired early in life than when they are acquired later. In line with certain picture naming studies that have failed to find reliable effects of word frequency when AoA was also taken into consideration (Barry et al., 2001; Bonin et al., 2002), we did not find a reliable contribution of objective word frequency, nor did it interact significantly with AoA. Clearly, the identification of the conditions that lead to the observation of both word frequency and AoA effects in picture naming is an issue that requires further investigation. We did not find that either Fam or VC made a significant contribution in predicting naming times. However, these variables have not been found to contribute robustly to object naming times (Barry et al., 1997; Bonin et al., 2002; Cycowicz et al., 1997; Ellis \& Morrison, 1998; Jolicœur, 1985). Finally, we did not find Imag, a variable which has often been considered to index the semantic level, to be a significant predictor of action naming latencies. This finding is surprising because, as was noted in the introduction, certain studies suggest that semantic representations corresponding to actions are sparser than those corresponding to objects (Gentner, 1981; Masterson \& Druks, 1998). Therefore, one might have been led to predict that Imag should play an important role in action naming. The lack of an effect of this variable might be related to its lower variance in the present data set.

The observation that the determinants that have been identified as reliably influencing object naming performance are similar to those found in action naming strongly suggests that action naming involves levels of processing similar to those that have been identified in object naming. However, although the main predictors of naming

Table 5

Values of Multiple $\boldsymbol{R}$ and Beta Weights for the Independent Variables in Spoken and Written Picture Naming

\begin{tabular}{|c|c|c|c|c|c|c|c|c|}
\hline \multirow[b]{3}{*}{ Multiple $R$} & \multicolumn{4}{|c|}{ Spoken Picture Naming } & \multicolumn{4}{|c|}{ Written Picture Naming } \\
\hline & \multicolumn{4}{|c|}{0.675} & \multicolumn{4}{|c|}{0.741} \\
\hline & $\beta$ & $S E$ & $t$ & $p$ & $\beta$ & $S E$ & $t$ & $p$ \\
\hline AoA & .306 & .112 & 2.74 & .01 & 247 & .106 & 2.32 & .02 \\
\hline NA $(H)$ & .232 & .086 & 2.69 & .01 & .271 & .077 & 3.50 & .001 \\
\hline IA & -.462 & .092 & -5.03 & .001 & -.485 & .085 & -5.72 & .001 \\
\hline $\mathrm{VC}$ & 141 & .095 & 1.48 & .14 & .144 & .085 & 1.69 & .09 \\
\hline Cum. 1 & -.059 & .080 & -0.74 & .46 & 167 & .101 & 1.64 & .10 \\
\hline Imag & -.064 & .090 & -0.71 & .48 & -.119 & .083 & -1.43 & .16 \\
\hline Fam & -.044 & .102 & -0.43 & .67 & -.005 & .087 & -0.06 & .95 \\
\hline Let & & & & & .230 & .073 & 3.16 & .001 \\
\hline Phons & .033 & .110 & .30 & .76 & & & & \\
\hline Dur & .017 & .089 & .20 & .85 & .012 & .082 & .15 & .88 \\
\hline
\end{tabular}

Note-AoA, estimated age of acquisition; NA, name agreement $(H$, statistic $H$ ); IA, image agreement; VC, visual complexity; Cum. freq., cumulative word frequency (log transformed) of the modal verb from LEXIQUE (Frantext; New et al., 2001); Imag, imageability; Fam, conceptual familiarity; Let, number of letters; Phons, number of phonemes; Dur, duration. 
Table 6

Descriptive Statistics Corresponding to Action and Object (Spoken and Written) Naming Times

\begin{tabular}{|c|c|c|c|c|c|c|c|c|c|c|}
\hline & \multicolumn{5}{|c|}{ Actions } & \multicolumn{5}{|c|}{ Objects } \\
\hline & $N$ & $M$ & $S D$ & Min & Max & $N$ & $M$ & $S D$ & Min & Max \\
\hline \multicolumn{11}{|c|}{ Spoken Naming } \\
\hline RT1 & 141 & 1,594 & 422 & 889 & 2 & 232 & 1,1 & 291 & 729 & 3,633 \\
\hline RT2 & 141 & 1,479 & 391 & 852 & 3,362 & 230 & 1,059 & 286 & 728 & 3,633 \\
\hline RT3 & 112 & 1,397 & 308 & 852 & 2,360 & 203 & 1,009 & 182 & 728 & 1,697 \\
\hline \multicolumn{11}{|c|}{ Written Naming } \\
\hline RT1 & 142 & 2,389 & 674 & 1,366 & 4,774 & 232 & 1,428 & 350 & 992 & 3,216 \\
\hline RT2 & 142 & 2,204 & 597 & 1,355 & 4,315 & 232 & 1,374 & 321 & 976 & 3,216 \\
\hline RT3 & 116 & 2,071 & 477 & 1,355 & 4,315 & 199 & 1,312 & 248 & 976 & 2,580 \\
\hline \multicolumn{11}{|c|}{$\begin{array}{l}\text { Note- }-N \text {, number of observations; } S D \text {, standard deviation; RT } 1 \text {, no re- } \\
\text { striction of items on the basis of the NA score and no trimming of the } \\
\text { latency data; RT } 2 \text {, naming latencies greater than two standard devia- } \\
\text { tions from the item means excluded; RT3, the same criteria as defined } \\
\text { for RT2 plus exclusion of all items for which there is less than } 50 \% \text { of } \\
\text { correct responses. }\end{array}$} \\
\hline
\end{tabular}

latency appear to be the same in object and action naming, naming difficulty seems to be different for objects and actions. The analyses reported above have shown that verbs have lower NA scores (in terms of \%NA) and a higher number of alternative names than do nouns. This suggests that actions are more difficult to name than are objects, in part because the mapping from meaning to label is more variable for verbs than for nouns. In other words, there are generally more ways to depict an action verbally than to depict an object.

The difficulty of naming actions as compared with objects can also be indexed by naming times. As can be seen from Table 6, action naming latencies are longer than object naming latencies. Whatever the criteria applied to analyze the data (when only items with a correct response rate of at least $50 \%$ are included and a trimming procedure is applied or when the full set of items is used and no trimming procedure is applied), actions still take longer to be named than objects do in both writing and speaking. In order to explore further the action-object naming time difference, we conducted two different kinds of analyses. In the first set of analyses, we performed a post hoc a priori matching of object and action items on rated AoA, NA, Imag, log frequency, IA, Fam, and number of letters. Owing to the stringent selection conditions, $\mathrm{VC}$ and number of phonemes were not controlled for a priori and were therefore introduced as covariates in analyses of covariance, with the nature of the items (action vs. object) taken as an independent variable and naming times as a dependent variable (spoken and written naming times, respectively). The results were clearcut: For both speaking and writing, the time difference between the naming of actions and objects was large and reliable $(p<$ $.01)$. Multiple regression analyses were subsequently performed on the spoken and written naming latencies with the same set of independent variables as those described for the multiple regression analyses reported above. However, the nature of the items was introduced as a dichotomous variable. The main findings, as described above, were again found in relation to the continuous independent variables (NA, IA, and AoA were found to be reliable determinants of naming speed in both speaking and writing). Importantly, the dichotomous variable was a strong and reliable determinant of naming speed in both production modes, as Székely et al. (in press) have found in spoken naming in English. Taken together, the two studies indicate that the naming time difference between actions and objects persists even when several important dimensions, which differentiate the two types of stimuli, are controlled for. The issue that remains is to determine why action naming is a slower and, therefore, more difficult process than is object naming. One possibility is that action naming is more difficult than object naming because actions demand more conceptual processing than do objects. Székely et al. introduced a measure of conceptual complexity that corresponds to the number of relevant objects or protagonists in each stimulus. However, the action-object naming latency difference was still reliable. As was acknowledged by Székely et al., this is a somewhat rough measure of conceptual complexity. One possibility put forward by Székely et al. is that action naming requires a greater degree of "scene parsing"a than is typically required for object naming. In the same way as those designed for objects, the development of on-line measures of action identification would help to determine whether the action-object naming latency difference is, partly or fully, attributable to differences acting at the identification level involved in both action and object naming. $\mathrm{Fu}$ ture work will now have to identify the key factor(s) underlying the action-object naming speed difference.

\section{REFERENCES}

Alario, F. X., \& Ferrand, L. (1999). A set of 400 pictures standardized for French: Norms for name agreement, image agreement, familiarity, visual complexity, image variability, and age of acquisition. Behavior Research Methods, Instruments, \& Computers, 31, 531552 .

Barry, C., Hirsh, K. W., Johnston, R. A., \& Williams, C. L. (2001). Age of acquisition, word frequency, and the locus of repetition priming of picture naming. Journal of Memory \& Language, 44, 350-373.

BARRY, C., Morrison, C. M., \& Ellis, A. W. (1997). Naming the Snodgrass and Vanderwart pictures: Effects of age of acquisition, frequency, and name agreement. Quarterly Journal of Experimental Psychology, 50A, 560-585.

Berman, S., Friedman, D., Hamberger, M., \& Snodgrass, J. G. (1989). Developmental picture norms: Relationships between name agreement, familiarity, and visual complexity for child and adult ratings of two sets of line drawings. Behavior Research Methods, Instruments, \& Computers, 21, 371-382.

Berndt, R. S., Mitchum, C. C., Haendiges, A. N., \& Sandson, J. (1997). Verb retrieval in aphasia: 1. Characterizing single word impairments. Brain \& Language, 56, 68-108.

BIRD, H., HowARD, D., \& FRANKLIN, S. (2001). Noun-verb differences? A question of semantics: A response to Shapiro and Caramazza. Brain \& Language, 76, 213-222.

Bonin, P., Chalard, M., MÉOT, A., \& FAyol, M. (2002). The determinants of spoken and written picture naming latencies. British Journal of Psychology, 93, 89-114.

BONIN, P., \& FAYOL, M. (2000). Writing words from pictures: What rep- 
resentations are activated and when? Memory \& Cognition, 28, 677689

Bonin, P., FAYOL, M., \& Chalard, M. (2001). Age of acquisition and word frequency in written picture naming. Quarterly Journal of Experimental Psychology, 54A, 469-489.

Bonin, P., MÉot, A., Aubert, L., Malardier, N., Niedenthal, P., \& CAPElle-ToczeK, M.-C. (2003). Normes de concrétude, de valeur d'imagerie, de fréquence subjective et de valence émotionnelle pour 866 mots [Concreteness, imageability, subjective frequency and emotional valence norms for a set of 866 French words]. L'Année Psychologique, 104, 655-694.

Bonin, P., Peereman, R., Malardier, N., Méot, A., \& Chalard, M. (2003). A new set of 299 pictures for psycholinguistic studies: French norms for name agreement, image agreement, conceptual familiarity, visual complexity, image variability, age of acquisition, and naming latencies. Behavior Research Methods, Instruments, \& Computers, 35, 158-167.

Caramazza, A. (1997). How many levels of processing are there in lexical access? Cognitive Neuropsychology, 14, 177-208.

CARAmazza, A., \& Hillis, A. E. (1991). Lexical organization of nouns and verbs in the brain. Nature, 349, 788-790.

CARroll, J. B., \& White, M. N. (1973). Word frequency and age of acquisition as determiners of picture-naming latency. Quarterly Journal of Experimental Psychology, 25, 85-95.

Chalard, M., Bonin, P., Méot, A., Boyer B., \& Fayol, M. (2003). Objective age-of-acquisition (AoA) norms for a set of 230 object names in French: Relationships with other variables used in psycholinguistic experiments, the English data from Morrison et al. (1997) and naming latencies. European Journal of Cognitive Psychology, 15, 209-245.

Cochran, W. G. (1952). The $\chi^{2}$ test of goodness of fit. Annals of Mathematical Statistics, 23, 315-345.

Cohen, J., MacWhinney, B., Flatt, M., \& Provost, J. (1993). PsyScope: An interactive graphic system for designing and controlling experiments in the psychology laboratory using Macintosh computers. Behavior Research Methods, Instruments, \& Computers, 25, 257-271.

Cuetos, F., \& Alija, M. (2003). Normative data and naming times for action pictures. Behavior Research Methods, Instruments, \& Computers, 35, 168-177.

Cuetos, F., Ellis, A.W., \& Alvarez, B. (1999). Naming times for the Snodgrass and Vanderwart pictures in Spanish. Behavior Research Methods, Instruments, \& Computers, 31, 650-658.

Cycowicz, Y. M., Friedman, D., Rothstein, M., \& Snodgrass, J. G. (1997). Picture naming by young children: Norms for name agreement, familiarity, and visual complexity. Journal of Experimental Child Psychology, 65, 171-237.

DAmasio, A. R., \& TRANEL, D. (1993). Nouns and verbs are retrieved with differently distributed neural systems. Proceedings of the $\mathrm{Na}$ tional Academy of Sciences, 90, 4957-4960.

Daniele, A., Giustolisi, I., Silveri, M. C., Colosimo, C., \& Gainotti, G. (1994). Evidence for a possible neuroanatomical basis for lexical processing of nouns and verbs. Neuropsychologia, 32, 1325-1341.

Dell, G. S., \& O'Seaghdha, P. G. (1992). Stages of lexical access in language production. Cognition, 42, 287-314.

Dell, G. S., Schwartz, M. F., Martin, N., Saffran, E. M., \& GAGNON, D. A. (1997). Lexical access in aphasic and nonaphasic speakers. Psychological Review, 104, 801-838.

Dell'ACQuA, R., LotTo, L., \& JoB, R. (2000). Naming times and standardized norms for the Italian PD/DPSS set of 266 pictures: Direct comparisons with American, English, French, and Spanish published databases. Behavior Research Methods, Instruments, \& Computers, 32, 588-615.

Denes, G., \& Dalla Barba, G. (1998). G. B. Vico, precursor of cognitive neuropsychology? The first reported case of noun-verb dissociation following brain damage. Brain \& Language, 62, 29-33.

Ellis, A. W., \& MORRISON, C. M. (1998). Real age-of-acquisition effects in lexical retrieval. Journal of Experimental Psychology: Learning, Memory, \& Cognition, 24, 515-523.

FiEZ, J. A., \& TRANEL, D. (1997). Standardized stimuli and procedures for investigating the retrieval of lexical and conceptual knowledge for actions. Memory \& Cognition, 25, 543-569.
GENTNER, D. (1981). Some interesting differences between verbs and nouns. Cognitive \& Brain Theory, 4, 161-178.

Gilhooly, K. J., \& Gilhooly, M. L. (1979). Age-of-acquisition effects in lexical and episodic memory tasks. Memory \& Cognition, 7, 214223.

HumPhre Ys, G. W., RidDoch, M. J., \& QuinLAN, P. T. (1988). Cascade processes in picture identification. Cognitive Neuropsychology, $\mathbf{5}$, 67-103.

JESCHENIAK, J. D., \& LEVELT, W. J. M. (1994). Word frequency effects in speech production: Retrieval of syntactic information and of phonological forms. Journal of Experimental Psychology: Learning, Memory, \& Cognition, 20, 824-843.

JOLICÄUR, P. (1985). The time to name disoriented natural objects. Memory \& Cognition, 13, 289-303.

LACHMAN, R. (1973). Uncertainty effects on time to access the internal lexicon. Journal of Experimental Psychology, 99, 199-208.

LaChMAN, R., ShafFer, J. P., \& HenRiKus, D. (1974). Language and cognition: Effects of stimulus codability, name-word frequency, and age of acquisition on lexical reaction time. Journal of Verbal Learning \& Verbal Behavior, 13, 613-625.

LEVELT, W. J. M. (1989). Speaking: From intention to articulation. Cambridge, MA: MIT Press.

Levelt, W. J. M., Roelofs, A., \& Meyer, A. S. (1999). A theory of lexical access in speech production. Behavioral \& Brain Sciences, 22, 175.

MARTEIN, R. (1995). Norms for name and concept agreement, familiarity, visual complexity and image agreement on a set of 216 pictures. Psychologica Belgica, 35, 205-225.

MASTERSon, J., \& DRUKS, J. (1998). Description of a set of 164 nouns and 102 verbs matched for printed word frequency, familiarity and age-of-acquisition. Journal of Neurolinguistics, 11, 331-354.

MCCARTHY, R., \& WARRINGTON, E. K. (1985). Category specificity in an agrammatic patient: The relative impairment of verb retrieval and comprehension. Neuropsychologia, 23, 709-727.

Miceli, G., Silveri, C., Nocentini, U., \& Caramazza, A. (1988). Patterns of dissociation in comprehension and production of nouns and verbs. Aphasiology, 2, 351-358.

Molfese, D. L., Burger-Judisch, L. M., Gill, L. A., Golinkoff, R. M., \& HIRSCH-PASEK, K. A. (1996). Electrophysiological correlates of noun-verb processing in adults. Brain \& Language, 54, 388413.

Morrison, C. M., ChAppell, T. D., \& Ellis, A. W. (1997). Age of acquisition norms for a large set of object names and their relation to adult estimates and other variables. Quarterly Journal of Experimental Psychology, 50A, 528-559.

Morrison, C. M., Ellis, A. W., \& Quinlan, P. T. (1992). Age of acquisition, not word frequency, affects object naming, not object recognition. Memory \& Cognition, 20, 705-714.

New, B., Pallier, C., Ferrand, L., \& Matos, R. (2001). Une base de données lexicales du français contemporain sur internet: LEXIQUE [A contemporary lexical database for French on the internet: LEXIQUE]. L'Année Psychologique, 101, 447-462. Available at http://www.lexique.org.

OldFIELD, R. C., \& WingField, A. (1965). Response latencies in naming objects. Quarterly Journal of Experimental Psychology, 17, 273281

Paivio, A., Clark, J. M., Digdon, N., \& Bons, T. (1989). Referential processing: Reciprocity and correlates of naming and imaging. Memory \& Cognition, 17, 163-174.

Pind, J., Jonsdottir, H., Tryggvadottir,H. B., \& Jonsson, F. (2000). Icelandic norms for the Snodgrass and Vanderwart (1980) pictures: Name and image agreement, familiarity, and age of acquisition. Scandinavian Journal of Psychology, 41, 41-48.

ROELOFS, A. (2000). WEAVER ++ and other computational models of lemma retrieval and word-form encoding. In L. Wheeldon (Ed.), Aspects of speech production (pp. 71-114). Sussex, U.K.: Psychology Press.

Sanfeliu, M. C., \& Fernandez, A. (1996). A set of 254 SnodgrassVanderwart pictures standardized for Spanish: Norms for name agreement, image agreement, familiarity, and visual complexity. $B e$ havior Research Methods, Instruments, \& Computers, 28, 537-555. SHAPIRO, K., \& CARAMAZZA, A. (2001a). Language is more than its 
parts: A reply to Bird, Howard, and Franklin (2001). Brain \& Language, 78, 397-401.

ShaPIRo, K., \& CARAMAZZA, A. (2001b). Sometimes a noun is just a noun: Comments on Bird, Howard, and Franklin (2000). Brain \& Language, 76, 202-212.

SNODGRASS, J. C., \& VANDERWART, M. (1980). A standardized set of 260 pictures: Norms for name agreement, image agreement, familiarity, and visual complexity. Journal of Experimental Psychology: Human Learning \& Memory, 6, 174-215.

SNODGRASS, J. G., \& YUdITSKY, T. (1996). Naming times for the Snodgrass and Vanderwart pictures. Behavior Research Methods, Instruments, \& Computers, 28, 516-536.

Székely, A., D’Amico, S., Devescovi, A., Federmeier, K., Herron, D., Iyer, G., Jacobsen, T., ArÉvalo, A. L., VArgha, A., \& BATES, E. (in press). Timed action and object naming. Cortex.

SzéKely, A., D' Amico, S., Devescovi, A., Federmeier, K., HerRON, D., IYeR, G., JACOBSEN, T., \& BATES, E. (2003). Timed picture naming: Extended norms and validation against previous studies. $B e$ havior Research Methods, Instruments, \& Computers, 35, 621-633.

TARDIF, T. (1996). Nouns are not always learned before verbs: Evidence from Mandarin speakers' early vocabularies. Developmental Psychology, 32, 492-504.

VITKOVITCH, M., \& TYRRELL,L. (1995). Sources of disagreement in object naming. Quarterly Journal of Experimental Psychology, 48A, 822-848.

\section{NOTES}

1. It is worthy of note that Lachman (1973; Lachman et al., 1974) used subjective frequency ratings as a measure of word frequency.

2.The formula given by Snodgrass and Vanderwart (1980) to compute the $H$ statistic is as follows:

$$
H=\sum_{i=1}^{k} p_{i} \log _{2}\left(1 / p_{i}\right),
$$

where $k$ is the number of different names given for each action picture and $p_{i}$ is the proportion of participants giving each name.

3. In order to respect Cochran's (1952) rules, the first two intervals $([0,10]$ and $[10,20])$ were collapsed.

4. We restricted our comparison to the objects taken from the Alario and Ferrand (1999) database.

5. It may be argued that strict reductions of the naming time data lead to findings that are restricted to a set of "easier" items. It should be noted, however, that the main findings reported below were also found when all the items were included (thus, whatever the NA scores) and no trimming procedure was applied.

(Manuscript received October 24, 2002; revision accepted for publication June 30, 2003.) 